\title{
LIGHT PROPAGATION IN LIQUID CRYSTAL FIBER STRUCTURES
}

\author{
T. NAsieowski AND T.R. WoLińskI \\ Faculty of Physics, Warsaw University of Technology \\ Koszykowa 75, 00-662 Warszawa, Poland
}

\begin{abstract}
The paper analyzes the light propagation in anisotropic liquid crystal fiber structures in order to find the possibilities to apply them to control and switch the polarization state and modulate some parameters of the transmitted light. The long term goal and motivation of the authors was the suggestion to replace the light modulating liquid crystal cell by a new generation of liquid crystal waveguides as an all-fiber light modulator, as well as sensors for different environmental parameters.
\end{abstract}

PACS numbers: 42.79.Hp, 42.70.Df, 42.81.Qb

\section{Introduction}

Since in the functional fiber optics there is a general tendency to replace all the optical bulk elements by the equivalent fiber-optic realizations, in this paper results of theoretical studies of light propagation in optical fibers with liquid crystalline elliptical-core have been reported.

The liquid crystalline-core optical fiber acts as an optically anisotropic medium characterized by an indices ellipsoid and can serve as a fiber with easily controlled birefringence. However, birefringence is equal to zero in a cylindrical-core fiber when the liquid crystal molecules are parallel to the fiber $z$ axis, and also this is a typical structure of liquid crystal inside the hollow-core fiber. For no other reason, it was decided to use elliptical-core liquid crystal fibers where the birefringence has always non-zero value due to the non-symmetrical geometry of the core.

\section{Theoretical analysis of light propagation in elliptical-core liquid crystal fibers}

The elliptical fiber is characterized by elliptical cross-section of its core. It has the property of guiding two different orthogonal polarized modes with a small difference of its propagation constants despite the isotropic material of the fiber 
core. Therefore the elliptical fiber propagates the light like an anisotropic fiber with circular cross-section. The difference between the propagation constants is called birefringence and it can be changed by many environmental conditions. However, we have much more possibilities of light propagation influencing or adapting this kind of fiber sensor element for a desired range, external conditions and others in the case of anisotropic material of elliptical fiber core. Well-known, widely used, relatively cheap, and with large abilities for adapting to the given conditions, anisotropic material is just liquid crystal.

In this paper the properties of light propagation in anisotropic elliptical and step index fibers are discussed. For the purpose of achieving a more simple form for analytical solution the nematic liquid crystals with three characteristic molecules structures are investigated. Nevertheless, before we describe the possibility of getting the analytical solution, the simple approximation method for fast assessment of the elliptical liquid crystal core fiber birefringence is shown in the following point.

\subsection{Approximate analysis of light propagation in elliptical-core liquid crystal fibers}

The preliminary investigation of induced birefringence in liquid crystal fibers can be realized with the use of the following formula precisely described in $[1,2]$

$$
B=\Delta^{2} F(e, V),
$$

where $\Delta$ is the normalized difference of refractive differences, $e$ is an ellipticity, $V$ is a normalized frequency and $F$ is a function.

Figure 1 was created according to Eq. (1) and it demonstrates the possibility of changing the birefringence in the fiber by altering the resultant refractive indices of the core. One can notice that while changing the difference between ordinary and extraordinary refractive indices of the liquid crystal in the fiber core the birefringence can change up to one order of magnitude, which is concerned as a relatively big change.

The dependence of the modes number on the resultant refractive index shown in Fig. 2 for two different radii of the circular liquid crystal core is very interesting. It proves that changes of environmental conditions influencing the resultant core

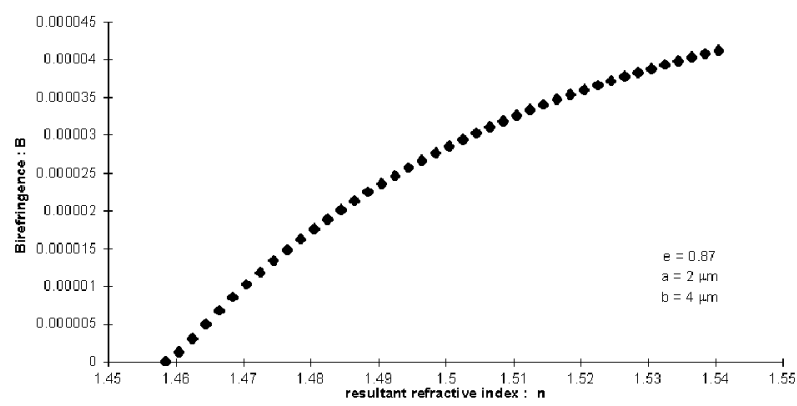

Fig. 1. Birefringence of liquid crystal fiber in a function of resultant refractive index of the fiber core for the given value of core ellipticity $e$ and its semi-axes $a$ and $b$. 


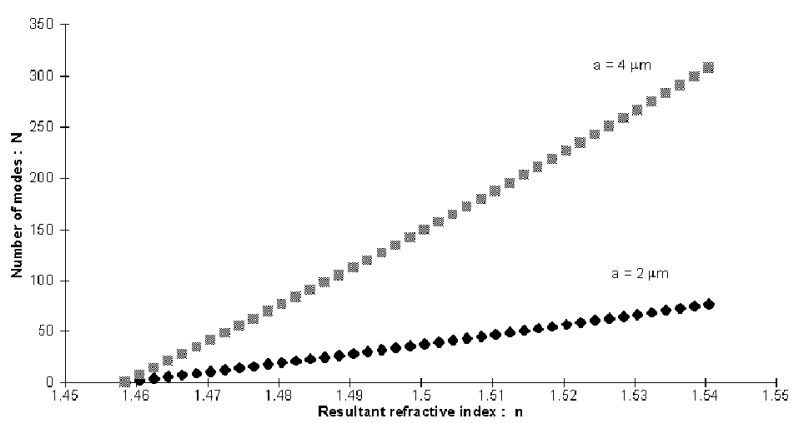

Fig. 2. Number of propagated modes in a function of resultant refractive index for two different radii of the circular core.

refractive index can increase or decrease the number of propagated modes. If this type of the liquid crystal fiber is placed just before the modal fiber coupler it would control the splitting ratio of this coupler by e.g. changing the external local electric field. It gives the opportunity to build the variable fiber coupler electrically controlled without any mechanical parts.

Such a simple model presented above in this part may be used for a fast and preliminary verification of the experiments, introductory prediction of the optical characteristics of liquid crystal and for matching them to the desired conditions. This method cannot help us to find the molecule structure or direction of the birefringence changes, the type of reorientation, and others. Generally this method does not describe precisely changes in the anisotropic core structure. To be able to find partially the more precise description a more complicated method should be derived. Most of the methods described in the literature, e.g. [3], assume weakly guiding conditions, but in our situation the difference between refractive indices of liquid crystal in the fiber core and its silica glass cladding are too large. The possible way to find this type of analytical solution is proposed in the next part of this chapter.

\subsection{Analytical solution of light propagation in elliptical-core liquid crystal fibers}

It is comfortable to analyze the elliptical liquid crystal fiber in elliptical coordinates system shown in Fig. 3. The elliptical coordinates system $(\xi, \eta, z)$ are represented by orthogonal to each other families of semi-focused elliptical and hyperbolic cylinders and moreover the coordinates $\xi$ and $\eta$ vary in the following ranges: $0 \leq \xi \leq \infty, 0 \leq \eta \leq 2 \pi$.

The relations between elliptical coordinates and Cartesian coordinates $(x, y, z)$ are the following:

$$
x=q \operatorname{ch} \xi \cos \eta, \quad y=q \operatorname{sh} \xi \sin \eta, \quad z=z .
$$

It is assumed that the fiber core is composed of an elliptical dielectric "cylinder" with the electrical permittivity tensor $\overline{\bar{\varepsilon}}$. Out of the surface $\xi=\xi_{0}$, i.e. out of the fiber core, we have an unlimited dielectric area called fiber cladding with the dielectric constant $\varepsilon_{2}$ (Fig. 4). Additionally we assume that the cladding is homogeneous, isotropic, and transparent for transmitted light, but the core is 


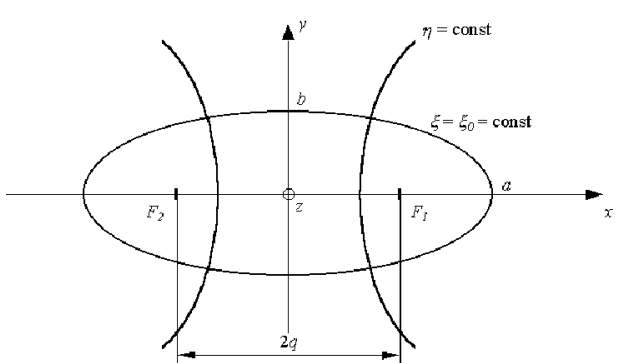

Fig. 3. Elliptical coordinates system. Ellipse $\xi_{0}$ describes the core-cladding border.

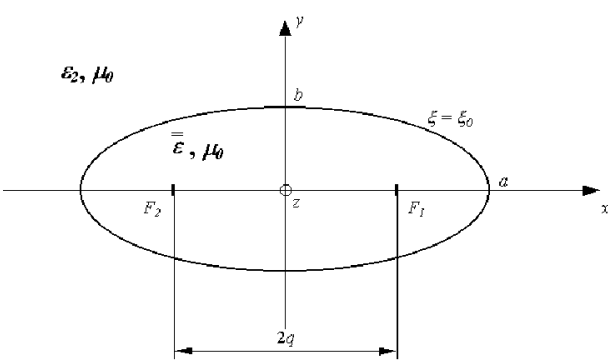

Fig. 4. Cross-section of the elliptical fiber: $a=q \operatorname{ch} \xi_{0}-\operatorname{long}$ semi-axis, $b=q \operatorname{sh} \xi_{0}-$ small semi-axis, $q$ - semi-focal length.

an anisotropic medium. Neither the media of core nor the cladding are magnetic media.

We are considering three characteristic nematic liquid crystal molecules structures in the waveguide core. Taking into account the Frank free energy density, also presented in $[4,5]$, which assumes that the orientation order of molecules is constant without changing the environmental parameters, we introduce the molecule structures in Fig. 5 and described below:

a) planar structure - molecules of liquid crystal lie parallel to the fiber long axis;

b) hyperbolic structure - molecules by their positions create the hyperbolic coordinates;

c) elliptical structure - molecules by their positions create the elliptical coordinates.

The planar structure is the most popular one, because during the filling process of the waveguide by liquid crystal the molecules tend to a parallel position to the glass surface. The hyperbolic structure can be created when the molecules are forced to be perpendicular to the glass surface. The elliptical structure is appearing especially when a constant electric field is applied along the long axis of the elliptical waveguide core. 


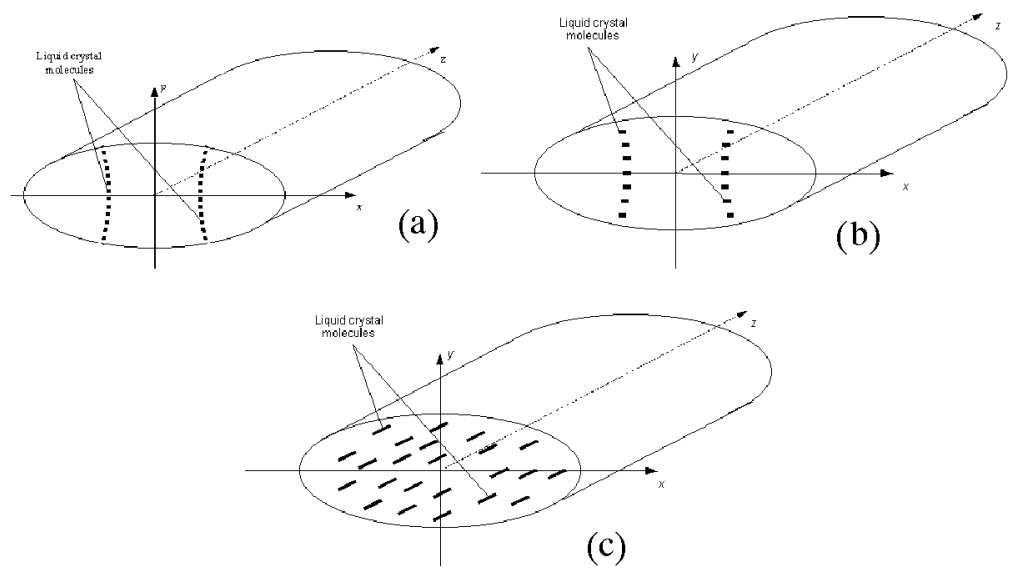

Fig. 5. Three characteristic molecule structures of liquid crystal in the elliptical fiber core, (a) hyperbolic, (b) elliptical, (c) planar.

All structures described above can be represented by the diagonal electrical permittivity tensor $\overline{\bar{\varepsilon}}$

$$
\overline{\bar{\varepsilon}}=\left[\begin{array}{ccc}
\varepsilon_{\xi} & 0 & 0 \\
0 & \varepsilon_{\eta} & 0 \\
0 & 0 & \varepsilon_{z}
\end{array}\right] .
$$

It is possible because an electrical permittivity tensor has to be symmetrical and the direction of the molecules is always parallel to one of the elliptical coordinate axes.

Then we have the following: for the planar structure $\varepsilon_{\xi}=\varepsilon_{\perp}$, $\varepsilon_{\eta}=\varepsilon_{\perp}$, $\varepsilon_{z}=\varepsilon_{\|}$, for the hyperbolic structure $\varepsilon_{\xi}=\varepsilon_{\perp}, \varepsilon_{\eta}=\varepsilon_{\|}, \varepsilon_{z}=\varepsilon_{\perp}$, and for the elliptical structure $\varepsilon_{\xi}=\varepsilon_{\|}, \varepsilon_{\eta}=\varepsilon_{\perp}, \varepsilon_{z}=\varepsilon_{\perp}$, where $\varepsilon_{\perp}, \varepsilon_{\|}$are dielectric constants respectively for the polarization of light perpendicular and parallel to the molecules, respectively.

The algorithm guiding to the characteristic equation is the following [6]:

A. Transform the electrical conductivity tensor into the elliptical coordinates system and its diagonal form;

B. Find the formulae for the longitudinal electromagnetic field components for desired modes, which have the solutions in non-modified and modified Mathieu functions of I, II, and III order [7];

C. Find the longitudinal electromagnetic field components in a function of the transversal components;

D. Determinant of the set of the equations, which can be drawn from boundary conditions for tangent components, should be equal zero, which gives the characteristic equation.

This method gives a possibility to find the analytical solution of light propagation in the elliptical waveguide with the liquid crystal anisotropic core. Thanks 
to this method it is possible to investigate the ideal and not disturbed liquid crystal molecule structure in the elliptical core. However, to be able to model the propagation of light in a waveguide with an arbitrary molecule structure, together with the method presented above the advanced numerical methods should be implemented.

The next chapter shortly describes how to experimentally investigate the liquid crystal fibers, how to change the structure of molecules and how this fiber could be used.

\section{Liquid crystal fiber light propagating devices}

Many different parameters modulating light propagation in liquid crystals have been intensively studied over the past years holding a great potential for applications to various kinds of sensors utilizing fiber optic sensing techniques. Any prospective liquid crystal sensors should be coupled to optical fibers that deliver optical signals to a sensing region.

Very interesting and different experiments can be proceeded with the liquid crystal elliptical-core fiber proposed in this paper. A special method for connecting the liquid crystal fiber with leading-in and leading-out fibers was developed. This method allows us to avoid fusion of the fibers, which could destroy the liquid crystal inside the sensing part. All the fiber sections: lead-in, liquid crystal and lead-out, can be glued inside an external capillary. From the point of view of optical power the best efficiency can be achieved by using the single mode lead-in fiber and the multimode lead-out fiber to receive the light transmitted by the sensing liquid-crystal fiber. The described configuration of the fibers is shown in Fig. 6.

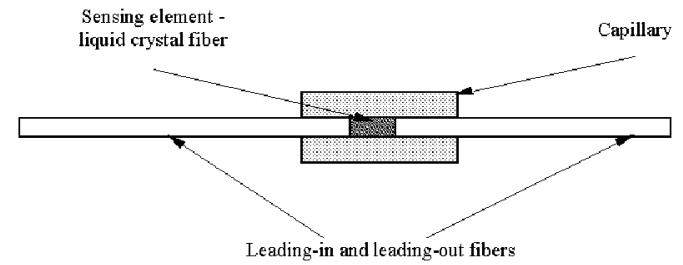

Fig. 6. Schema of the liquid crystal fiber sensor.

Some preliminary experiments were presented in [2], where one can find the example characteristics of light propagation in liquid crystal fibers under changes of different external conditions like temperature, electric field, and hydrostatic pressure.

\section{Conclusions and possibilities of implementations}

To conclude, two ways of theoretical analysis of light propagation in the elliptical-core liquid crystal fibers have been presented. The first one, a very rough method allows us to make some preliminary study and the second one is based on the analytical solution, which together with numerical methods can be a powerful tool. 
Theoretical analysis and some experiments [2] prove that it is possible to build very promising devices. Among these devices we can find the following:

- Controlled and adjustable fiber coupler without mechanical elements.

- Fiber sensor based on a nonlinear self-focusing effect - working between linear and nonlinear regime. For a given molecules structure we have the nonlinear phenomena called self-focusing described in [8]. In these conditions lots of optical power is coupled into the lead-out fiber. When the external conditions change the structure of the molecules inside the liquid crystal fiber and hence the conditions of nonlinear phenomena are not fulfilled any more and the lead-out fiber receives much less light.

- Polarimetric sensor with an active liquid crystal element also participated in a construction of optical neural network. Liquid crystal fiber can be also used as a switching element or a cell of the neural network. Also the polarization of transmitted light is different when the structure of molecules is changed by external conditions.

- Discrete and multi-threshold warning sensor. There are different phases of liquid crystals, which can be changed also by temperature. Each of the liquid crystal phases has different transmission conditions and while this phase is altered then the amount of light reaching the detector is different. In that way the sensor can warn us about overcoming certain limits induced by e.g. temperature.

\section{Acknowledgments}

One of the authors (T.N.) would like to acknowledge the financial help of NATO Research Fellowship.

\section{References}

[1] M. Varnham, D.N. Payne, A.J. Barlow, R.D. Birch, J. Lightwave Technol. 1, 332 (1983).

[2] T. Nasiłowski, T.R. Woliński, Acta Phys. Pol. 95, 767 (1999).

[3] K.S. Chiang, J. Lightwave Technol. LT-5, 737 (1987).

[4] H. Lin, P. Palffy-Muhoray, M.A. Lee, Mol. Cryst. Liq. Cryst. 204, 189 (1991).

[5] T.-J. Chen, S.-H. Chen, J. Lightwave Technol. 13, 1698 (1995).

[6] A. Majewski, Z. Krasiński, Numerical Analysis of Single-Mode Elliptical Fibers, Warsaw University of Technology, Warsaw 1991 (in Polish).

[7] M. Abramowitz, A. Stegun, Handbook of Mathematical Functions with Formulas, Graphs, and Mathematical Tables, National Bureau of Standart Applied Mathematics Series - 55, Washington (DC) 1964.

[8] M.A. Karpierz, M. Sierakowski, M. Świłło, T.R. Woliński, Mol. Cryst. Liq. Cryst. 320, 157 (1998). 